\title{
HYDRODYNAMIC PARTICLE MIGRATION IN A CONCENTRATED SUSPENSION UNDERGOING FLOW BETWEEN ROTATING ECCENTRIC CYLINDERS
}

Nhan Phan-Thien ${ }^{1}$, Alan L. Graham ${ }^{2}$, Stephen A. Altobelli ${ }^{3}$, James R. Abbott ${ }^{2}$, and Lisa A. Mondy ${ }^{4}$

${ }^{1}$ Mech. Eng. Dept., University of Sydney, Sydney, New South Wales 2006 (Australia)

${ }^{2}$ Los Alamos National Laboratory, Los Alamos, New Mexico 87545 (USA)

${ }^{3}$ Lovelace Medical Foundation, 2425 Ridgecrest Dr., S.E, Albuquerque, New Mexico 87108 (USA)

${ }^{4}$ Sandia National Laboratories, Albuquerque, New Mexico 87185 (USA)

\section{ABSTRACT}

We report on experimental measurements and numerical predictions of shear-induced migration of particles in concentrated suspensions subjected to flow in the wide gap between a rotating inner cylinder placed eccentrically within a fixed outer cylinder (a cylindrical bearing). The suspensions consists of large, noncolloidal spherical particles suspended in a viscous Newtonian liquid. Nuclear magnetic resonance (NMR) imaging is used to measure the time evolution of concentration and velocity profiles as the flow induces particle migration from the initial, well-mixed state.

A model, originally proposed by Phillips et al. (1992) is generalized to two dimensions. The coupled equations of motion and particle migration are solved numerically using an explicit pseudo-transient finite volume formulation. While not all of the qualitative features observed in the experiments are reproduced by this general numerical implementation, the velocity predictions show moderately good agreement with the experimental data.

\section{INTRODUCTION}

A number of experimental studies have determined that particles in initially well-mixed suspensions migrate to the low shear-rate region in nonhomogeneous shear flow fields (Karnis et al. 1966, Arp and Mason 1977, Gadala-Maria 1979, Gadala-Maria and Acrivos 1980, Leighton 1985, Hookham 


\section{DISCLAIMER}

Portions of this document may be illegible in electronic image products. Images are produced from the best available original document. 
1986, Graham et al. 1991, Abbott et al. 1991, Koh et al. 1994). This migration occurs even under conditions in which inertial and colloidal forces are usually considered negligible. Here, we report on the particle migration, observed with NMR imaging, in a wide-gap cylindrical bearing, with a rotating inner cylinder placed off the axis of a fixed outer cylinder. The flow in this bearing is two dimensional, as opposed to the one-dimensional flow studied previously in the wide gap between rotating coaxial cylinders (Abbott et al. 1991).

Stokes flow of a Newtonian (single phase) liquid in an infinite cylindrical bearing can be described analytically (Duffing 1924, Wannier 1950). Whereas the lowest shear rate displayed in an apparatus with coaxial cylinders occurs at the outer wall, the lowest shear rates in some cylindrical bearings do not occur along the wall. In these geometries, a reversed flow region exists in the wide part of the gap between the two cylinders. We will compare velocity profiles obtained in a concentrated suspension to those predicted for a Newtonian liquid undergoing flow in cylindrical bearings with two eccentricities. The suspension consists of relatively large, unimodal particles suspended in a viscous Newtonian liquid at a solids fraction of 0.50 . NMR images show that extensive particle migration occurs in these bearings and that this migration creates a region of maximum solids concentration away from the wall when reverse flow is present.

Other investigators have proposed mechanisms to model the suspended particle migration caused by the presence of nonuniform shear gradients (Phillips et al. 1992, Koch 1989, Leighton and Acrivos 1987, Nott and Brady 1994, Zhang and Acrivos 1994). Except for Zhang and Acrivos (1994), who studied the flow of nonneutrally buoyant particles in pipe flow, these models have been compared to data only from one-dimensional flows. Here, we compare the NMR data from the two-dimensional bearing flow to numerical predictions using a model expression previously used successfully to describe one-dimensional flows by Phillips et al. (1992). The model expression consists of two parts: a Newtonian constitutive equation in which the viscosity depends on the local solids volume fraction and a diffusion equation that accounts for shear-induced particle migration. We expand this expression to two dimensions by describing the flow in terms of the shear rate tensor $\mathbf{D}$ and the migration in terms of gradients in the generalized shear rate $\dot{\gamma}=\sqrt{2 \operatorname{trD}^{2}}$.

We solve the coupled equations of motion and particle migration using an explicit pseudo-transient finite volume technique. We find reasonable overall 
agreement between the model and data, although not all of the qualitative features observed in the experiments are reproduced by this numerical implementation.

In the next section we briefly describe the test fluids and experimental procedure. We then discuss the NMR imaging results in the third section. In the fourth, we describe a finite volume numerical technique, and in the fifth, we report the modeling results compared to data. We discuss and summarize the results in the final section.

\section{EXPERIMENTAL METHOD}

The techniques used in this experiment remained essentially the same as those described in Abbott et al. (1991). The suspensions consisted of $50 \%$ by volume of polymethyl methacrylate (PMMA) particles suspended in a density-matched liquid. This liquid was tested in a Rheometrics RMS 605 up to a shear rate of $100 \mathrm{~s}^{-1}$ and displayed no significant non-Newtonian behavior. The suspending liquid viscosity $\eta_{0}$ was $4.95 \mathrm{~Pa} \cdot \mathrm{s}$ at $23.15^{\circ} \mathrm{C}$. The particles were unimodal but somewhat polydisperse, with a mean diameter of approximately $675 \mu \mathrm{m}$ and a standard deviation in diameter of about $15 \%$. Although the temperature of each suspension was controlled only by the room air conditioner, no settling of the suspended particles was detected over the duration of a typical experiment.

Each apparatus consisted of a rotating solid PMMA cylinder eccentrically placed in a fixed outer PMMA tube capped with solid PMMA disks. The outer radius of the inner rod $\left(R_{i}\right)$ was $0.64 \mathrm{~cm}(1 / 4 \mathrm{in})$, the inner radius of the outer tube $\left(R_{o}\right)$ was $2.54 \mathrm{~cm}$ ( $\left.1 \mathrm{in}\right)$. The length of the suspension-filled cavity was $25 \mathrm{~cm}$. Figure 1 is a sketch of the cross-section of the device. The inner rod was placed at one of two positions, with the offset, $e$, of the axis of the inner rod either $0.64 \mathrm{~cm}(1 / 4 \mathrm{in})$ or $0.95 \mathrm{~cm}(3 / 8 \mathrm{in})$ off the axis of the fixed outer cylinder (Fig. 1). With these dimensions, the maximum offset possible $e_{\max }=R_{o}-R_{i}$ (occurring when the inner cylinder touches the outer) is $1.9 \mathrm{~cm}(3 / 4 \mathrm{in})$. The eccentricity ratio $e / e_{\max }$ is $1 / 3$ for the first apparatus and $1 / 2$ for the second.

In this set of experiments, the inner cylinder was rotated at a constant rate of approximately $90 \mathrm{rpm}$ by a motor. Care was taken not to turn the inner cylinder until initial NMR images of the initial state were taken. The motor was stopped after a predetermined number of rotations, and NMR images of 
the Couette were taken. The NMR technique is described by Abbott et al. (1991) and Graham et al. (1991).

Images were taken of a cross-sectional slice, 2.4-cm thick, perpendicular to the Couette axis, about midway along the apparatus. The time required for each image was $\sim 8 \mathrm{~min}$. Back-to-back repeated images indicate no significant settling or particle redistribution during this interval. In these experiments, the intensity values in the part of the slice corresponding to the suspensionfilled apparatus were normalized such that the average intensity of the image matched the liquid fraction, which was known to a high degree of accuracy.

\section{EXPERIMENTAL RESULTS}

Figure 2 shows the time progression of the NMR concentration images of the bearing with eccentricity ratio equal to $1 / 3$, from the initially well-mixed suspension to the steady state after 14,000 revolutions of the inner cylinder. One can see a dramatic change in solids concentration, with the steady-state solids concentration at the outer wall much higher than at the inner cylinder. This is quantified in Fig. 3a, in which the solid line is the NMR measurement of the liquid fraction (1- $\phi$ ) across the diameter of the apparatus bisecting the inner rod (the $\mathrm{x}$-axis of Fig. 1), at steady state. The abscissa represents the distance along the $\mathrm{x}$-axis with the origin at the center of the inner rod. This graph shows that the solids concentration is fairly close to maximum (random) packing at the outer wall, where the liquids fraction is about 0.4; whereas, the solids fraction is only about 0.27 (liquid fraction about 0.73 ) at the inner cylinder wall. This concentration change represents a dramatic change in suspension viscosity: the apparent viscosity of a homogeneous suspension of $60 \%$ of uniform spheres is about 25 times that of a suspension with a solids concentration of $27 \%$ (Rutgers 1962, Thomas 1965).

Figure $3 \mathrm{~b}$ shows the NMR measurements of velocity (solid line) across this same slice. The theoretical prediction (dashed line) for a Newtonian liquid is also shown for reference. In this geometry, there is no reverse flow for a Newtonian liquid (Wannier, 1950). The velocity in the suspension falls off much more quickly than in a Newtonian liquid, and is virtually zero about 1 $\mathrm{cm}$ from the inner shaft. The suspension is moving in approximately a circle about the inner shaft, more symmetrically than a Newtonian liquid would flow in the same geometry.

Figure 4 shows the concentration image of the bearing with eccentricity 
ratio of $1 / 2$ after over 10,000 revolutions of the inner shaft. Here, the maximum concentration is not at the outer wall. This is shown quantitatively in Fig. 5a, which is again a graph of the fluid fraction in a slice across the diameter of the apparatus bisecting the inner shaft. Within the widest portion of the gap, the solids concentration is almost to maximum packing; whereas, at the outer wall the solids concentration is close to the original value of 0.5. The solid line in Fig. 5b is the NMR measure of liquid velocity. A flow reversal is predicted for a Newtonian liquid in this geometry (Wannier 1950); however, the magnitude of the reversed velocity seen in a Newtonian liquid is about $1 \%$ of the velocity on the inner shaft and much smaller than can be distinguished with the NMR technique. That a flow reversal occurs in the suspension seems to be indicated by the presence of a high concentration region not at the outer wall but in a region within the gap. This was confirmed by visual observations of a tracer particle in the suspension and determined that indeed a region of very slow (of order $1 \mathrm{~mm} / \mathrm{minute}$ ) reverse flow occurred.

\section{FINITE VOLUME MODELING}

\subsection{Governing Equations}

In the model presented Phillips et al, the stress tensor is given by the generalized Newtonian constitutive equation:

$$
\boldsymbol{\sigma}=-P \mathbf{1}+\boldsymbol{\tau}, \quad \boldsymbol{\tau}=2 \eta(\phi) \mathbf{D}
$$

where $\boldsymbol{\sigma}$ is the total stress tensor, $P$ is the hydrostatic pressure, $\boldsymbol{\tau}$ is the extra stress, $\mathbf{D}=\frac{1}{2}\left(\nabla \mathbf{u}+\nabla \mathbf{u}^{T}\right)$ is the strain rate tensor, $\mathbf{u}$ is the velocity field, the superscript $T$ denotes the transpose operation, $\eta$ is the fluid velocity, which is assumed to depend only on the local volume fraction of the solid phase, $\phi$. Krieger's form for the viscosity function is chosen here (Krieger, 1972):

$$
\frac{\eta}{\eta_{0}}=\frac{1}{\left(1-\phi / \phi_{m}\right)^{\alpha}}
$$

where $\eta_{0}$ is the viscosity of the suspending liquid, $\phi_{m}$ is the maximum volume fraction, and $\alpha$ is a constant of the order 2. In this paper we assume $\phi_{m}=$ 0.68 and $\alpha=1.82$, the values suggested by Phillips et al. (1992). 
The governing equations are the usual conservation statements:

$$
\begin{gathered}
\nabla \cdot \mathbf{u}=0 \quad \text { (conservation of mass), } \\
\nabla \cdot \boldsymbol{\sigma}=\mathbf{0} \quad \text { (conservation of linear momentum), }
\end{gathered}
$$

where the fluid inertia has been omitted. To this set of equations we add the conservation of the solid phase, which takes the form

$$
\frac{\partial \phi}{\partial t}+\mathbf{u} \cdot \nabla \phi=\nabla \cdot \mathbf{N}
$$

where $\mathbf{N}$ is the flux of the solid phase, given by the model equation

$$
\mathbf{N}=K_{c} a^{2} \phi \nabla(\dot{\gamma} \phi)+K_{\eta} a^{2} \dot{\gamma} \phi^{2} \nabla(\ln \eta),
$$

consisting of a contribution from the collision between the particles and a contribution from the gradient of the viscosity. This set of governing equations is subjected to the usual no-slip and no-flux boundary conditions at a solid surface. The latter boundary condition is written as

$$
\mathbf{N} \cdot \mathbf{n}=0
$$

where $\mathbf{n}$ is the outward unit normal vector on the surface.

\subsection{Explicit Finite Volume Method}

The existence of third-order derivatives (gradients of gradients of shear rate) and the strong coupling between the volume fraction and the viscosity imply that the solution will be most sensitive to small changes in the volume fraction, especially in the regions of low shear rates. This is confirmed by our brief investigation of a finite element scheme using operator splitting techniques, which was proved to be quite unstable.

In this paper we employ a pseudo-transient finite volume scheme, which is based on the method of artificial compressibility due to Chorin (1967), where the continuity equation is replaced by

$$
\frac{\partial P}{\partial t}+\frac{1}{\delta} \nabla \cdot \mathbf{u}=0,
$$


where $\delta$ is the artificial compressibility, and $t$ is a time-like variable. Since we are interested in the steady-state solution $\delta$ is set to unity. The main idea behind this is that an unbalance in the mass conservation is used to drive the pressure correction. We may re-instate the time derivative $\rho \partial \mathbf{u} / \partial t$, with $\rho=1$, in the conservation of linear momentum and use the unbalance in the conservation of linear momentum to drive the velocity correction:

$$
\frac{\partial \mathbf{u}}{\partial t}=\nabla \cdot \boldsymbol{\sigma} .
$$

The computational domain is divided into non-overlapped finite volume cells, each of volume $V_{i}$ and bounding surface $S_{i}$. We now integrate the governing equations (5), (8-9) over this generic finite volume to obtain

$$
\begin{gathered}
\int_{V_{i}} \frac{\partial P}{\partial t} d V=-\int_{S_{i}} \mathbf{u} \cdot \mathbf{n} d S \\
\int_{V_{i}} \frac{\partial \mathbf{u}}{\partial t} d V=\int_{S_{i}} \boldsymbol{\sigma} \cdot \mathbf{n} d S
\end{gathered}
$$

and

$$
\int_{V_{i}} \frac{\partial \phi}{\partial t} d V=\int_{S_{i}}(\mathbf{N}-\mathbf{u} \phi) \cdot \mathbf{n} d S .
$$

In the simplest form of the finite volume method (but by far most commonly used in computational fluid dynamic problems) the field variables are assumed piece-wise constant. This is assumed here, leading to the discretized equations

$$
\begin{gathered}
V_{i} \frac{d P_{i}}{d t}=-\sum_{j}(\mathbf{u} \cdot \mathbf{n} S)_{j}, \\
V_{i} \frac{d \mathbf{u}_{i}}{d t}=\sum_{j}(\mathbf{t} S)_{j}, \\
V_{i} \frac{d P_{i}}{d t}=-\sum_{j}((\mathbf{N}-\mathbf{u} \phi) \cdot \mathbf{n} S)_{j},
\end{gathered}
$$

where $\mathbf{t}=\boldsymbol{\sigma} \cdot \mathbf{n}$ is the traction, the subscripted variable $i$ indicates the corresponding value for the cell $V_{i}$, and the sums are to be taken over all the bounding surfaces $S_{i}$ of cell $i$. In this paper, we are only concerned with twodimensional flows, in which the computational domains are discretized using 
unstructured triangular cells (Jin and Wiberg, 1990), an example of which is given in Fig. 6 (the volume of a cell is actually its area, and a bounding surface is actually a boundary line).

To calculate the integrations over the surface $S_{j}$ of $V_{i}$, we first evaluate the averaged values of the field variables on $S_{j}$, using corresponding values either side of the surface, between two neighboring cells sharing the same boundary, and then calculate the integrands using these averaged values. It can be shown that, on a two-dimensional grid patch consisting of equilateral triangles of the same size, the scheme is actually a central differencing scheme and is second-order accurate in space (Jameson and Mavriplis, 1986). If a bounding surface is also on the boundary of the domain, special consideration is required. On structured grids, we may extrapolate the values on the edge from the values in the neighboring cells. On unstructured grids, it is not obvious how to design an accurate extrapolation scheme. For simplicity, we adopt a zero-order approximation and set the values on such a surface equal to the values in the cell containing the surface.

Gradients of any quantity are calculated by assuming that they are constant within the cell, so that, for example

$$
(\nabla \phi)_{i}=\frac{1}{V_{i}} \int_{V_{i}} \nabla \phi d V=\frac{1}{V_{i}} \int_{S_{i}} \phi \mathbf{n} d V=\frac{1}{V_{i}} \sum_{j}(\phi \mathbf{n} S)_{j} .
$$

The set of ordinary differential equations in the time-like variable $t$ can be written in the form

$$
\frac{d}{d t} \mathbf{U}=\mathbf{F}(\mathbf{U})
$$

where $\mathrm{U}$ represents all the unknowns, $\left\{\mathbf{u}_{i}, P_{i}, \phi_{i}\right\}$. This set is integrated in $t$ using an adaptive fifth-order Runge-Kutta scheme. We can accelerate the convergence to the final steady-state solution by replacing $V_{i}$ on the left hand sides of (13-15) by a scale value $V_{c}$ (typically about the size of the smallest volume). In this way disturbances are expected to sweep a large cell in about the same number of iterations as with small cells. To obtain quantitative information on how far away from steady state the current solution is, we use the maximum residual

$$
\epsilon=\max _{i}\|\mathrm{~F}(\mathrm{U})\| \text {. }
$$

When this is less than a prescribed tolerance, of $O\left(10^{-6}\right)$, then the solution is deemed to be at steady state. 
Boundary conditions are easily accommodated in this scheme (no-slip, no-flux, or even traction boundary conditions), since the prescribed velocity, flux or traction can be directly fed into the right hand sides of (13-15). This explicit finite volume scheme has been used for solving some difficult problems in rheology, including the stick-slip problem of the upper-convected Maxwell model (Jin et al., 1990).

\section{NUMERICAL RESULTS}

\subsection{Circular Couette Flow}

We first benchmarked the code in the circular Couette flow, where analytical solution (Phillips et al., 1992) is available through solving the following equations

$$
\left(\frac{r}{R_{i}}\right)^{2}=\frac{\phi}{\phi_{w}}\left(\frac{1-\phi_{w} / \phi_{m}}{1-\phi / \phi_{m}}\right)^{\alpha\left(K_{\eta} / K_{c}-1\right)}
$$

and

$$
\frac{d}{d r}\left(\frac{u_{\theta}}{r}\right)=\frac{C}{r^{2}}\left(1-\frac{\phi}{\phi_{m}}\right)^{\alpha},
$$

subjected to the boundary conditions

$$
u_{\theta}\left(R_{i}\right)=\omega R_{i}, \quad u_{\theta}\left(R_{o}\right)=0,
$$

and

$$
\frac{R_{o}^{2}}{R_{o}^{2}-R_{i}^{2}} \int_{R_{i}}^{R_{o}} \phi(r) d r=\phi_{a} .
$$

In these equations, $R_{i}$ is the radius of the inner cylinder, which rotates with an angular velocity of $\omega, R_{o}$ is the radius of the stationary outer cylinder, $C$ is a constant to be determined from the boundary conditions, and $\phi_{a}$ is the prescribed average volume fraction of the solid phase, which is constant at all time. The values for the parameters $K_{\eta}$ and $K_{c}$ are the same as those used by Phillips et al. (1992).

A comparison between the numerical results, obtained with a mesh of 4084 elements, and the analytical solution for the concentration and the velocity profiles is given in Fig. 7. It is clear that we have a good agreement with the exact solution. In fact, the convergence to the exact solution is 
linear in the mesh size. With the adaptive integration scheme, the number of time steps required to achieve a steady state is of the order 10000 (30 minutes CPU time on a DecStation 5000/300), which is an order of magnitude fewer time steps than are necessary if a constant time step is used. The time step itself varies, depending on the estimated local error in each finite volume cell, but is not allowed to be greater than one. The scheme is pseudo-transient, and therefore the total time taken to reach steady state (about 6000, or about 1000 turns of the inner cylinder) does not have any direct physical relevance; but it does give an indication on how long it would take to reach steady state and is comparable to the 4000 turns which is necessary to reach an experimental steady-state (Abbott et al., 1991).

\subsection{Eccentric Bearing Configuration}

We now consider the flow in an eccentric bearing configuration with an eccentricity ratio of $1 / 2$, and with $R_{o}=1, R_{i}=0.25$, corresponding to one of the experimental setups; the computational mesh is displayed in Fig. 6. Initially the volume fraction is uniform at $50 \%$, and the inner cylinder is allowed to rotate. The kinematics quickly achieve a steady state after some 10000 time steps, as evidenced from the contours of velocities in Fig. 8. The volume fraction takes longer to reach steady state (about 20000 time steps); as the inner cylinder rotates, the solid phase is swept into long thin strips, migrating towards the outer cylinder forming a large essentially stagnant region in the wide gap region, with a correspondingly high solid volume fraction (about $62 \%$ ).

The steady state contours of the concentration are displayed in Fig. 9, as a three-dimensional map. In the figure, the computational mesh is elevated along the $z$-axis by an amount proportional to the volume fraction. In this way, one can visually determine if there is any local maximum, and assess the quality of the steady state of the solution by the degree of symmetry of the flow field.

The concentration and the velocity profiles along the narrow and the wide gaps are given in Fig. 5. Along the narrow gap, the concentration profile varies nearly linearly, from a low value of about $17 \%$ at the inner cylinder to a high value of about $45 \%$ at the outer cylinder. A similar trend is found along the wide gap, except that the concentration is more quadratic, varying between $18 \%$ and $62 \%$. Overall the concentration along any radial line 
increases monotonically, from a low value at the inner cylinder, to a high value at the outer cylinder, as seen in Fig. 9. Although the overall agreement with the data (as shown in Fig. 5) is fair, we find no recirculation at this eccentricity ratio, in contrast to the experimental observation of a tracer particle, mentioned above. (Keep in mind, however, that this recirculation velocity is very small, approximately $1 \%$ of the velocity at the shaft, and is well below the detection limit of the NMR measurements.) Allowing the solvent viscosity to be shear-thinning will not bring about a recirculation, because near the outer cylinder the low shear rate would reinforcing the noflow condition there due to the higher viscosity. Perhaps some amount of anisotropy, which is missing in the present model equations, would help. Finally, although recirculation is not predicted by the numerical results, the concentration profile is reasonably well-predicted (as seen from Fig. 5a) considering the uncertainty in the experimental data, and the simplicity of the model theory.

\section{SUMMARY}

NMR imaging allows the study of flow-induced particle migration in concentrated suspensions. Concentration profiles are easily determined, and good spatial resolution can be obtained. When subjected to flow between rotating eccentric cylinders, neutrally buoyant particles migrate away from the rotating inner rod. Depending on the geometry, the final region of highest solids concentration can either be at the outer wall or in a region within the gap.

We have modeled the suspension following the technique presented by Phillips et al. (1992). Here, we expanded the model expression to twodimensions by describing the flow in terms of the strain rate tensor and the migration in terms of gradients in the generalized shear rate. Although this expression resulted in particle migration and final concentrations that were approximately those measured with NMR imaging, important qualitative features of the flow could not be reproduced. Specifically, the flow reversal was not seen in the $1 / 2$ eccentricity ratio case and neither was the occurrence within the gap of the region of highest particle concentration along the line separating the recirculation region from the rest of the cylindrical bearing. What is obviously missing in the present model is the anisotropy that must be ever-present in suspensions. An improved model should have some degree 
of anisotropy, reflecting the differing behavior of the microstructure in shear and elongational flows.

Finally, the explicit finite volume scheme has been found very robust in this problem, although there are several third-order derivative terms, which tend to de-stabilize more sophisticated finite element methods. The use of the artificial compressibility method is a convenient technique for correcting the pressure; however transient flows cannot be treated by the method. We are currently investigate different finite volume schemes to overcome this problem.

\section{ACKNOWLEDGMENTS}

The authors would like to thank Nick Tetlow for his help in making the apparatuses used in this study and to Dr. Moshe Gottlieb and Dr. Tom Stephens for testing the density matched liquid. This work was sponsored by the U.S. Department of Energy at Los Alamos National Laboratory under Contract W-7405-ENG-36 with the University of California, and at Sandia National Laboratories under Contract DE-AC04-94-AL85000. The authors gratefully acknowledge partial support for this work by the U.S. Department of Energy, Division of Engineering and Geosciences, Office of Basic Energy Sciences and through Dr. R. S. Miller's section of the Mechanics Division, Office of Naval Research. Work at Lovelace Medical Foundation was partially supported by the Whitaker Foundation and the donors of the Petroleum Research Fund administered by the ACS.

\section{REFERENCES}

1. Abbott, J. R.; Tetlow, N.; Graham, A. L.; Altobelli, S. A.; Fukushima, E.; Mondy, L. A. and Stephens, T. S. J. Rheo., 1991, 35, 773.

2. Arp, P. A. and Mason, S. G. J. Colloid Interface Sci., 1977, 6144.

3. Chorin, A. J. J. Comp. Phys., 1967, 2, 12.

4. Duffing, G. Z. angew Math. Mech., 1924, 4, 296.

5. Gadala-Maria, F., PhD thesis, Stanford University, 1979.

6. Gadala-Maria, F. and Acrivos, A. J. Rheo., 1980, 24, 799. 
7. Graham, A. L.; Altobelli, S. A.; Fukushima, E.; Mondy, L. A. and Stephens, T. S. J. Rheo., 1991, 35, 191.

8. Hookham, P., PhD thesis, California Institute of Technology, 1986.

9. Jameson, A. and Mavriplis, D. A.I.A.A. J., 1986, 24, 611.

10. Jin, H.; Phan-Thien, N. and Tanner, R. I. Comp. Mech., 1990, 13, 433.

11. Jin, H. and Wiberg, N.-E. Int. I. Num. Methods Eng. , 1990, 29, 1501.

12. Karnis, A.; Goldsmith, H. L. and Mason, S. G., J. Colloid Interface Sci., 1966, 22, 531.

13. Koch, D. K. Phys. Fluids A, 1989, 1, 1742.

14. Koh, C. J.; Hookham, P. and Leal, L. G. . J. Fluid Mech., 1994, 266, 1.

15. Krieger, I. M. Adv. Colloid Interface Sci., 1972, 3, 111.

16. Leighton, D. T. PhD thesis, Stanford University, 1985.

17. Leighton, D. and Acrivos, A. J. Fluid Mech., 1987, 181, 415.

18. Nott, P. R. an Brady, J. F. J. Fluid Mech., 1994, 275, 155.

19. Phillips, R.J.; Armstrong, R.C.; Brown, R.A.; Graham, A. L. and Abbott, J.R., Phys. Fluids $A, \mathbf{1 9 9 2}, 4,30$.

20. Rutgers, R. Rheol. Acta, 1962, 16, 202.

21. Thomas, D. G. J. Colloid Sci., 1965, 20, 267.

22. Wannier, G. H. Quart. Appl. Math., 1950, $8,1$.

23. Zhang, K. and Acrivos, A. Int. J. Multiphase Flow, 1994, 20, 579.

\section{DISCLAIMER}

This report was prepared as an account of work sponsored by an agency of the United States Government. Neither the United States Government nor any agency thereof, nor any of their employees, makes any warranty, express or implied, or assumes any legal liability or responsibility for the accuracy, completeness, or usefulness of any information, apparatus, product, or process disclosed, or represents that its use would not infringe privately owned rights. Reference herein to any specific commercial product, process, or service by trade name, trademark, manufacturer, or otherwise does not necessarily constitute or imply its endorsement, recommendation, or favoring by the United States Government or any agency thereof. The views and opinions of authors expressed herein do not necessarily state or reflect those of the United States Government or any agency thereof. 
Figure Captions

Fig. 1: Cylindrical bearing geometry. The $\mathrm{x}$-axis passes through the center of both cylinders. The two cylinders are off-set by the distance $e$.

Fig. 2: NMR images of a bearing with an eccentricity ratio equal to $1 / 3$. The four figures show the initially well-mixed concentration profile followed by the concentration profiles after 200,800 and 14000 revolutions of the inner rod. The false color image uses yellow to highlight regions of high liquid (or low solids) concentration.

Fig. 3: The steady-state velocity and concentration profiles along the $\mathrm{x}$ axis of a cylindrical bearing with an eccentricity ratio equal to $1 / 3$. The abscissa represents a nondimensional distance across the $\mathrm{x}$-axis with the origin at the center of the inner rod. a) The solid concentration $\phi$ increases monotonically from a low at the inner cylinder wall. b) The velocity in the suspension (solid line) decreases more rapidly than the Newtonian velocity (dashed line) in the same geometry. Because the particle concentration is not uniform, the viscosity of the suspension is lower near the inner cylinder leading to higher shear rates than would be observed with a Newtonian fluid.

Fig. 4: NMR image of a bearing with an eccentricity ratio equal to $1 / 2$. Darker regions represent higher liquid fraction (lower solids fraction). The highest solids fraction occurs along a line within the widest portion of the gap.

Fig. 5: The steady-state velocity and concentration profiles along the $\mathrm{x}$ axis of a cylindrical bearing with an eccentricity ratio equal to $1 / 2$. The abscissa represents a nondimensional distance across the $\mathrm{x}$-axis with the origin at the center of the inner rod. a) The experimentally measured liquid concentration (solid line) decreases almost linearly from the inner cylinder to the outer cylinder along the narrow part of the gap. However along the wide part of the gap, the liquid fraction reaches a minimum (solids concentration reaches a maximum) at about $\mathrm{x}=1.1$. This probably represents a stagnant region in the flow. The numerical results (- - )predict a monotonic change with the maximum concentration at the out cylinder. b) Here, as with an eccentricity ratio of $1 / 3$, the velocity in the suspension (solid line) decreases more rapidly than the Newtonian velocity (Wannier 1950, - -) in the same geometry. Good agreement with our numerical results $(--)$ are shown.

Fig. 6: Numerical mesh demonstrating the use of unstructured triangular cells. For visualization, a less dense example mesh is shown.

Fig. 7: Numerical results (square points) compared with the analytical 
solution (solid line) for a suspension in a circular Couette flow.

Fig. 8: Steady-state velocity contours predicted numerically for a cylindrical bearing, with an eccentricity ratio equal to $1 / 2$, filled with a suspension with an overall volume fraction of solids of 0.50 . Steady-state values are reached after 10000 time steps.

Fig. 9: The steady-state concentration profiles predicted numerically for a cylindrical bearing, with an eccentricity ratio equal to $1 / 2$, filled with a suspension with an overall volume fraction of solids of 0.50 . Concentration profiles stabilize after 20000 time steps. Here the $\mathrm{z}$-axis represents the relative magnitude of the concentration. 


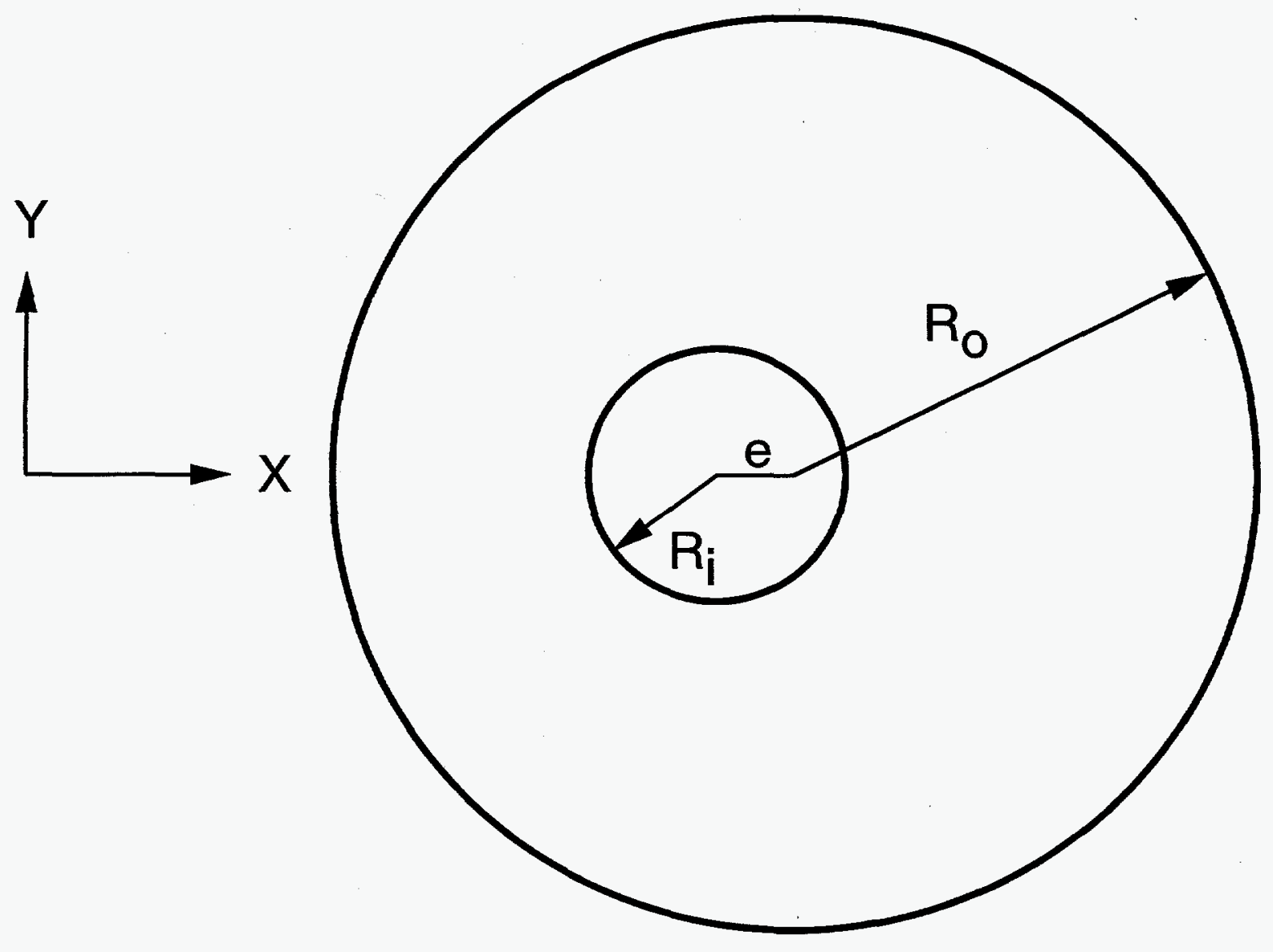

$F i$ 


\section{1}

0

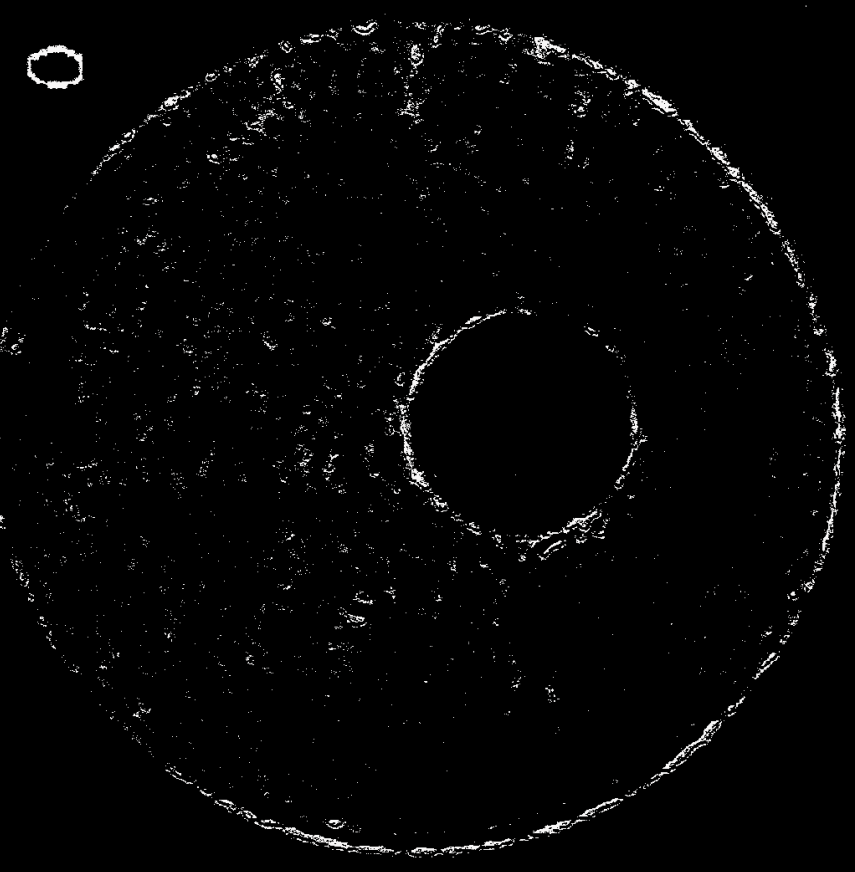

+8
8
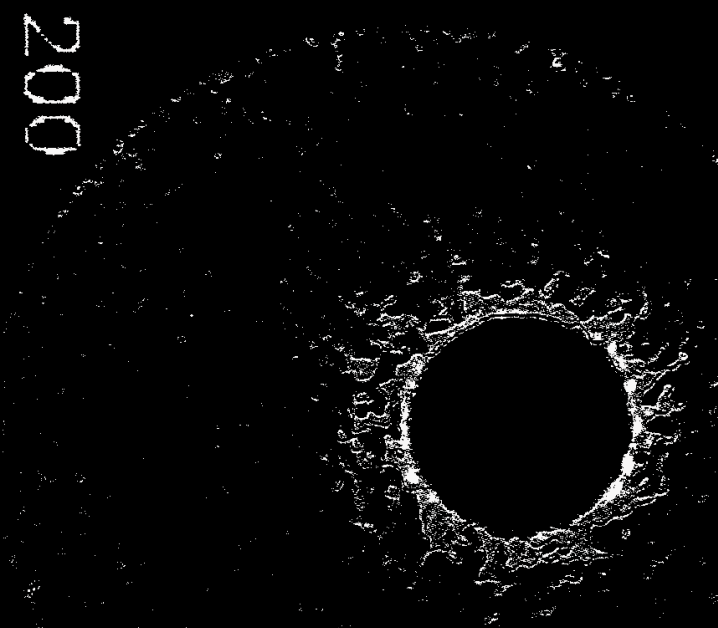

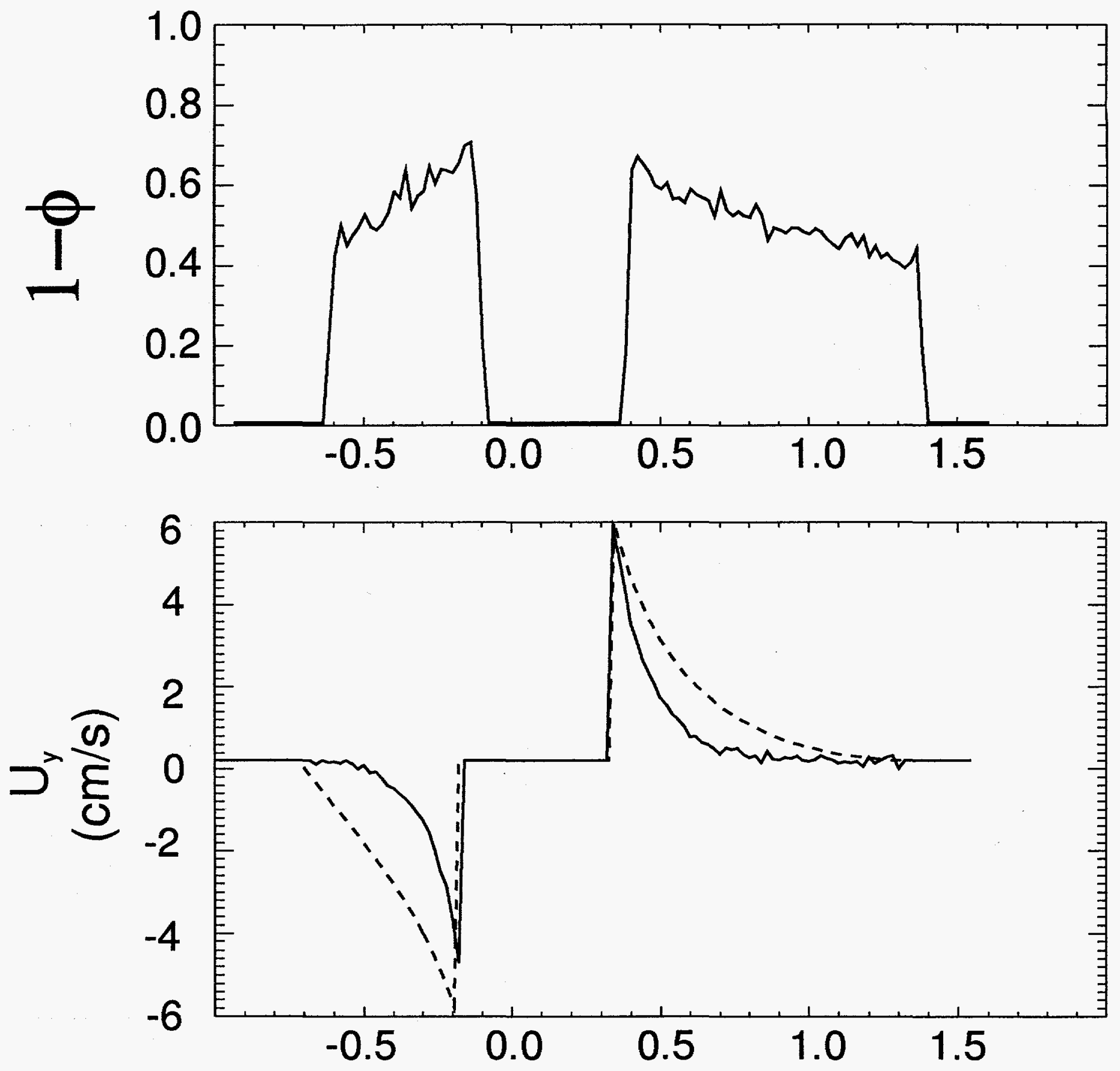

Fic $3 a, b$ 


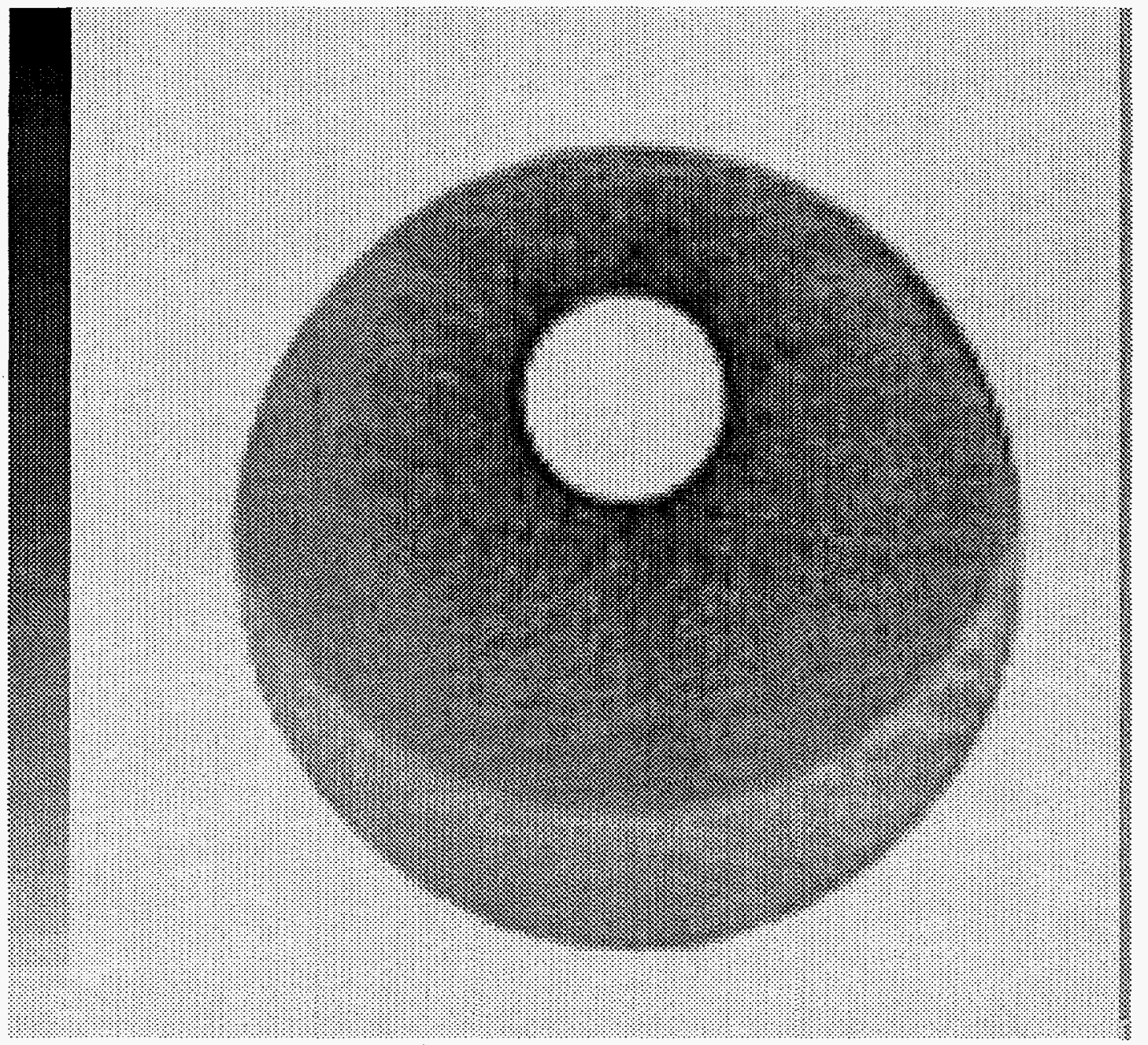

Fig 4 

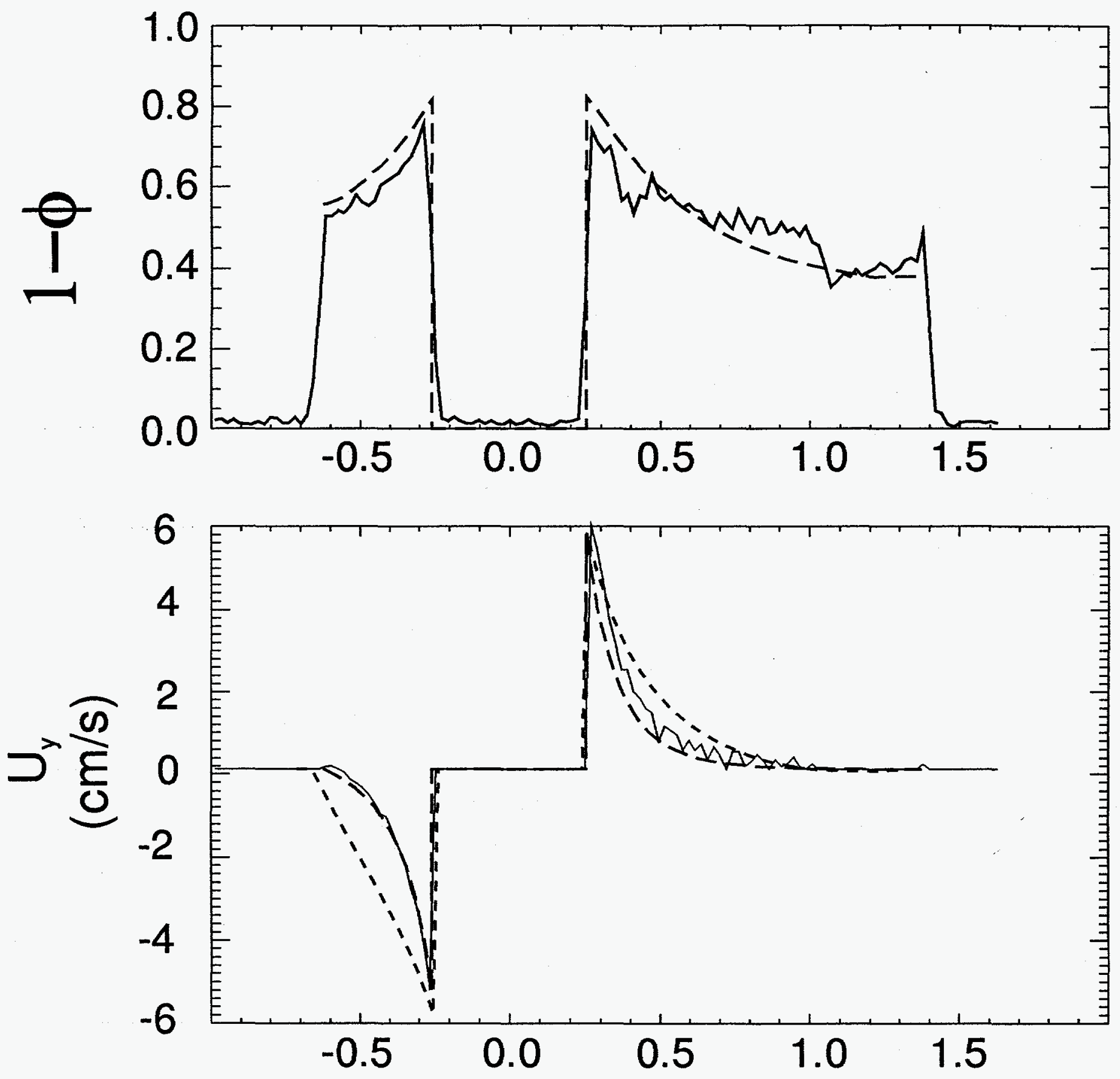

Fig $5 a, b$ 


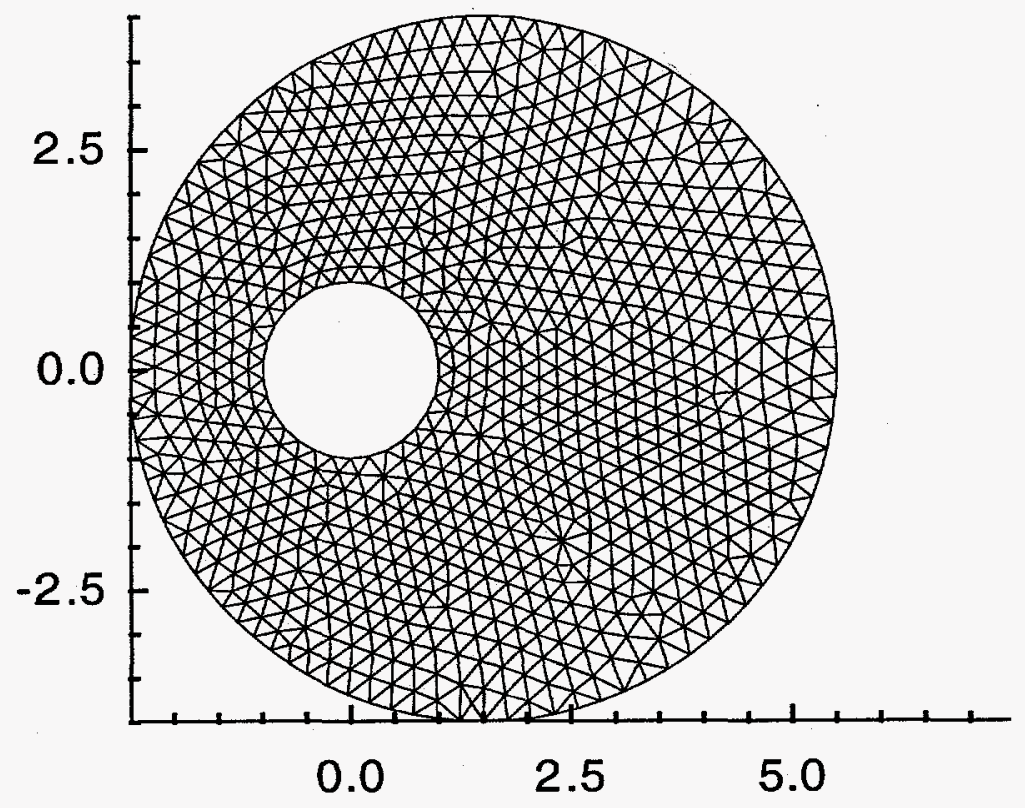

Fig $\$ 6$ 


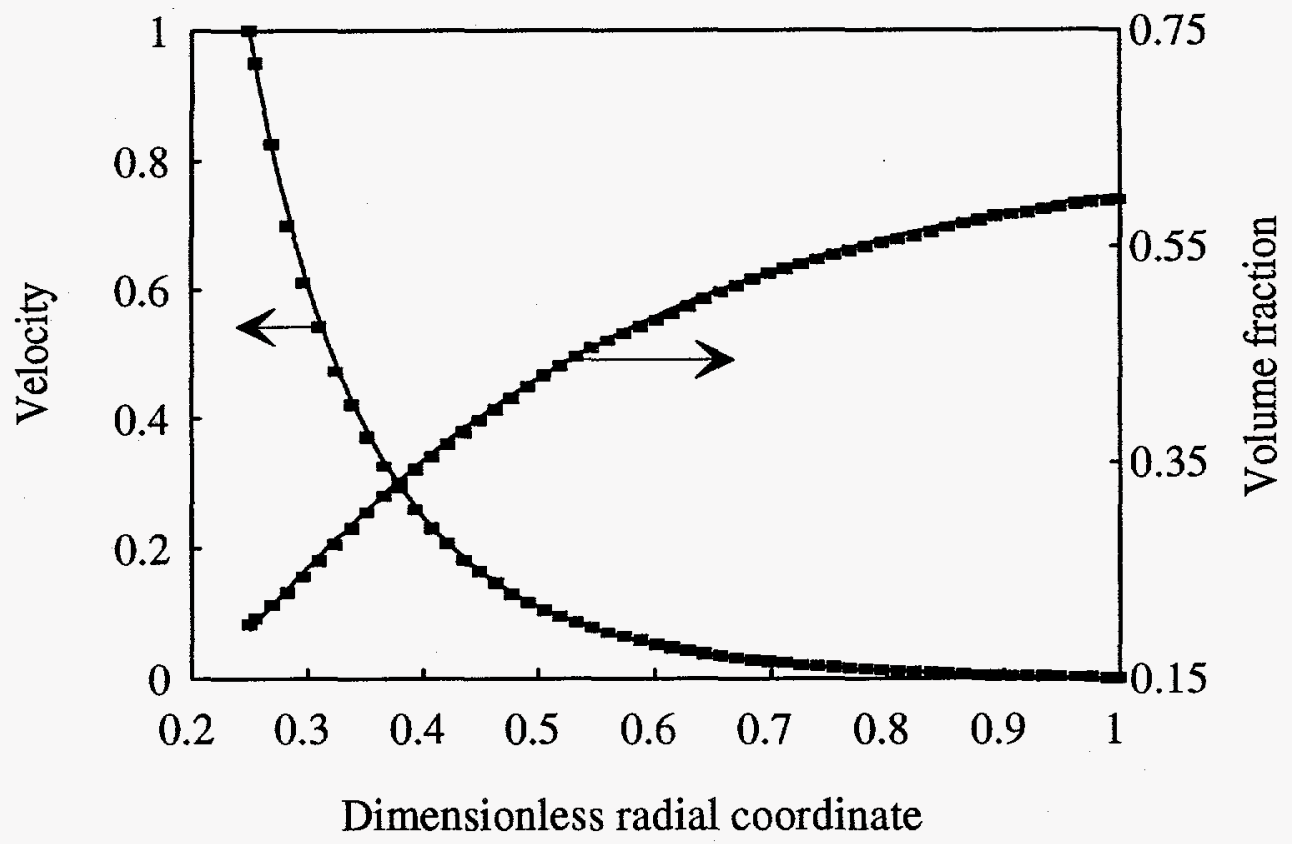

$F i g \& 7$ 

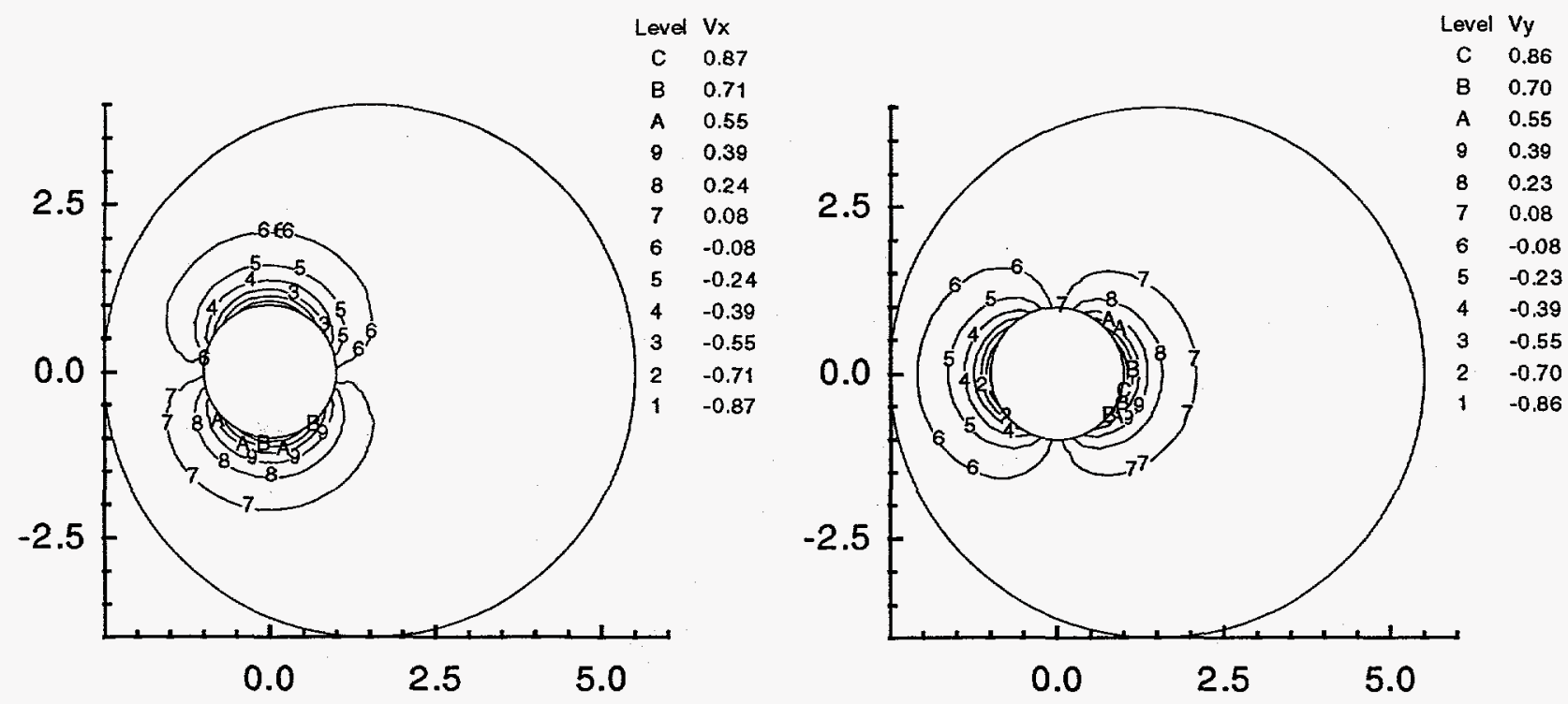

Fig 8 


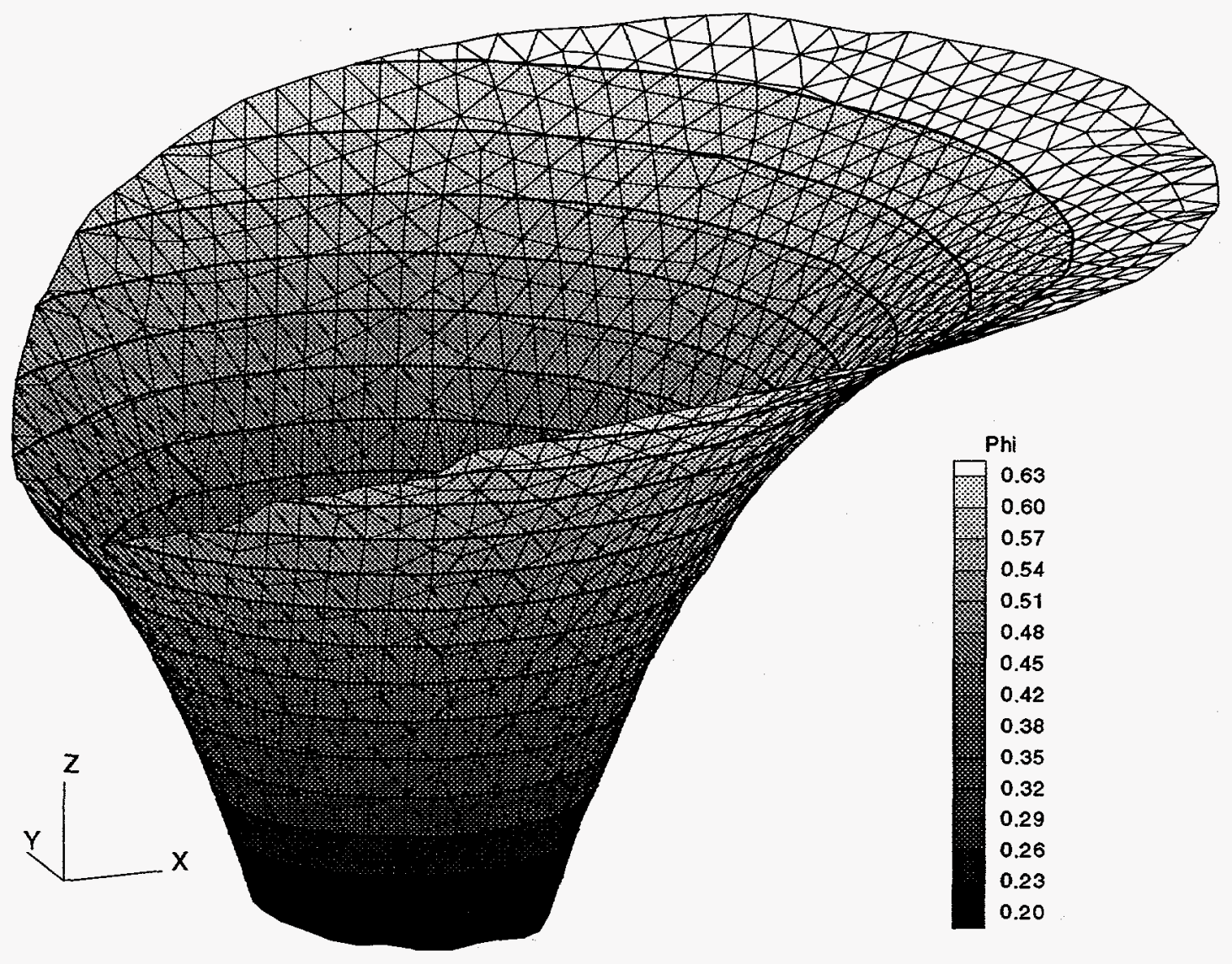

Fig te 9 